\title{
Crecimiento económico y el crédito bancario: un análisis de causalidad para México
}

\author{
Economic growth and bank credit: \\ a causality analysis for Mexico
}

\author{
José Luis de la Cruz Gallegos \\ y José Ángel Alcántara Lizárraga \\ ITSEM, campus Estado de México, México \\ jldg@itesm.mx \\ maquina_1065@hotmail.com
}




\section{Crecimiento económico y el crédito bancario: un análisis de causalidad para México}

\section{RESUMEN}

Se estima la relación que existe entre el sistema bancario y el crecimiento económico en México. Para ello se considera el crédito otorgado por los bancos al sector privado. Con el objeto de establecer las relaciones causales entre las variables se elaboran vectores autorregresivos y de cointegración. Los resultados muestran que prevalece una relación de causalidad entre el crédito y el crecimiento total, así como una interrelación bidireccional de este último con el financiamiento al consumo. De igual manera prevalece una relación entre el crédito al consumo y el crecimiento del sector terciario de la economía. Para los otros tipos de financiamiento no se encuentran resultados estadísticamente significativos, lo cual señala que el crédito bancario no impulsa el desarrollo de los sectores primarios y secundarios de la economía.

Palabras clave: cointegración, causalidad, crédito al consumo, mercado interno, auge de crédito

\section{Clasificación JEL: C32, G21, $\mathrm{O}_{4}$}




\title{
Economic growth and bank credit: a causality analysis for Mexico
}

\begin{abstract}
The present analysis estimates the relation between the banking system and economic growth. Therefore, it considers the credit granted by banks to the private sector. With the aim of establishing (in order to establish) causal relations among the variables, autoregressive and cointegration vectors are developed. The results show that it prevails a causal link between the credit and total growth as well as a bidirectional interrelation of the latter with the consumption financing. Similarly (In the same way), it prevails a relation between the credit designated for consumption and the tertiary economic sector growth. For the other types of financing there are not statistically significant results, which point out that banking credit does not boost the development of the primary and secondary sectors of the economy.
\end{abstract}

Keywords: cointegration, causality, consumption credit, domestic market, credit boom.

JEL Clasification: C32, G21, 047

Revista de Economía, vol. XXVIII, núm. 77, julio-diciembre de 2011/13-38 



\section{INTRODUCCIÓN}

En 1911 Joseph Schumpeter planteó la posibilidad de que el desarrollo del sistema financiero, entendido como la canalización del ahorro y la facilidad para realizar las transacciones financieras, sea un factor primordial en el proceso de innovación tecnológica y el crecimiento económico. King y Levine (1993) retoman esta idea mediante un estudio econométrico que contempló a 80 países en un periodo de casi 30 años. Los resultados obtenidos señalaron la presencia de una correlación positiva entre el desarrollo del sistema financiero y las tasas de crecimiento económico de largo plazo. De igual manera, su análisis refleja un impacto positivo que va del desarrollo del sistema financiero hacia la inversión y la productividad. Su tesis central acerca de los mecanismos de transmisión de dichos efectos fue que el crecimiento se consigue por medio de dos vías: a) la acumulación de capital físico y, b) la eficiencia en su distribución.

Los resultados del trabajo de King y Levine (1993) son trascendentales en el sentido de que muestran uno de los factores clave para el crecimiento de los países en vías de desarrollo y que se encuentran vinculados con la idea de la destrucción creativa de Schumpeter. La implicación económica fundamental radica en que la innovación es el motor del desarrollo: se destruyen viejos esquemas y modelos de negocio para crear nuevas herramientas que aumentan la productividad del "emprendedor innovador", lo cual, a su vez, redunda en mayores tasas de actividad económica. En este proceso, la correcta canalización de los recursos financieros hacia toda la economía es fundamental, pero es particularmente relevante en el impulso que dichos flujos pueden proporcionar a los sectores productivos motores del crecimiento y la innovación. En términos generales, la hipótesis central que se tiene respecto al desarrollo del sistema financiero gira en torno a que si el crédito llega a los diferentes sectores productivos, ello generará un vigoroso crecimiento.

En el presente análisis se busca establecer si lo anterior es válido para el caso de la economía mexicana y el crédito que otorga la banca privada. En teoría, las instituciones de crédito deberían constituirse en la principal fuente de financiamiento de las empresas y ocupar un papel preponderante en el ciclo de negocios del país. Sin embargo, la poca experiencia de los bancos privatizados durante la década de los años noventa y la 
ausencia de una adecuada regulación provocaron un aumento del crédito que terminó con la crisis bancaria de 1994-1995. A partir de esa fecha la evolución del crédito ha sido irregular tanto en la cantidad de recursos canalizados como en lo referente a los sectores productivos a los que se ha dirigido. Derivado de lo anterior, estimar los efectos de dicha estrategia es fundamental, especialmente para observar si el sistema de crédito tiene el impacto positivo sobre el crecimiento de México, en particular por la falta de cumplimiento de la hipótesis del ingreso permanente (HIP), así como por la relación que existe entre los denominados booms de crédito y las crisis económicas de los años noventa, elemento esencial para entender los eventos de expansión y recesión que han ocurrido en el país.

No obstante la extensa literatura teórica y empírica en el ámbito internacional sobre la relación que guarda el sistema financiero con el crecimiento, son pocos los estudios que existen para el caso concreto de México. Algunos han dado cuenta de una relación causal bidireccional positiva entre las variables (Rodríguez y López, 2009), aunque no existe evidencia de los canales de transmisión precisos a partir de los cuales el sistema financiero mexicano impacta en la generación de una mayor actividad económica. En contraste, otros trabajos empíricos como el de Tinoco, Torres y Venegas (2008) apuntan a que existe un efecto inverso entre la represión financiera y el crecimiento económico, así como una relación causal unidireccional entre el desarrollo financiero y el crecimiento económico.

La investigación sobre el desarrollo financiero y la actividad económica en México no es, pues, concluyente. De hecho, la teoría empírica internacional dista mucho de ser homogénea, por lo cual resulta de vital importancia presentar un análisis causal más desagregado para conocer los alcances del financiamiento en el país.

Es igualmente relevante citar que, de no existir evidencia suficiente que permita garantizar la existencia de efectos positivos del financiamiento hacia el crecimiento, se tendría que la intermediación bancaria no sería garante para favorecer que el ahorro de la economía se transforme en factor de desarrollo. Por lo anterior, el objetivo central del presente análisis es determinar si existen relaciones de causalidad entre el crédito otorgado por los bancos y los principales sectores de actividad económica del país. De igual manera, se busca establecer si dichos vínculos son de largo plazo. Los vectores autorregresivos (VAR) y de corrección de error (VEC) constituyen 
una metodología apropiada para establecer si existen interrelaciones causales en el sentido de Granger entre las variables bajo estudio y donde los VEC se aplican en caso de que exista una relación de cointegración.

El resto del trabajo se divide como sigue: en la sección dos se hace una revisión de la literatura sobre el tema, en la tres se reseña el caso mexicano en concreto, en la siguiente exponemos la metodología y los resultados, finalizamos con la presentación de las conclusiones.

\section{REVISIÓN DE LA LITERATURA}

A partir de la idea de Shumpeter (1911) se ha extendido el interés por estudiar los efectos que la estructura del sistema financiero tiene sobre la economía. En lo correspondiente a los modelos teóricos de crecimiento económico, en general se planteaba la profundidad financiera como un elemento exógeno, de forma que se creaba una limitante en el poder explicativo de dicha variable. Para superar esa restricción, Pagano (1993) propone un modelo de crecimiento endógeno tipo AK en el cual se tiene la tasa de crecimiento como una función del ahorro y de la productividad marginal social de la inversión. Por su parte, Bencivenga y Smith (1991) plantean un modelo en el que concluyen que la represión financiera, entendida como el establecimiento de techos en las tasas de interés y aumentos en los requerimientos de reservas, puede ser dañina para la economía al mermar la tasa de crecimiento de largo plazo. Stiglitz (1998) también considera que reprimir el sistema financiero para extraer rentas del sector privado, con el fin de financiar déficits públicos, puede ser muy perjudicial para la economía. El autor señala que el sistema financiero, entendido como el conjunto de bancos, otros intermediarios financieros, los mercados de deuda y de capital resuelven tres problemas fundamentales: la aglomeración de capital, su distribución en los usos más importantes y el monitoreo de que dichos recursos sean utilizados eficientemente. Asimismo, plantea la hipótesis de que los sistemas financieros que funcionan bien realizan un excelente trabajo de selección de los receptores más productivos y se aseguran de que éstos lo utilicen en las actividades que generan un mayor valor agregado en la economía. Finalmente, concluye que la inversión no únicamente depende de las tasas de interés, ya que factores 
como la investigación y desarrollo, el capital humano y la eficiencia de los procesos administrativos de las empresas son esenciales para determinar la magnitud de dicha variable.

Bajo este esquema, Tirado (2000) elabora un modelo cuya hipótesis básica consiste en que en una economía innovadora con bancos y dinero (este último no súper neutral) se puede alcanzar un PIB mayor vía el desarrollo del sector bancario. Se supone que las firmas en la economía son innovadoras, lo que causa crecimiento económico mediante la canalización del crédito al sector tecnológico. Dada una función de utilidad sujeta a restricciones presupuestarias monetarias y al considerar que la demanda de créditos por parte del sector innovador es igual a los planes de inversión en capital físico, se puede encontrar que, en una economía de mercado, la tasa de crecimiento depende positivamente de la productividad de los factores empleados en los procesos de innovación, de la calidad del capital humano y de los depósitos bancarios existentes en la economía. El modelo de Tirado (2000) plantea de manera precisa la existencia de una tasa de crecimiento vinculada de forma directa con la participación del sector financiero, vía el sector bancario.

Los autores Rodríguez y López (2009) resumen las visiones sobre la relación entre el sistema financiero y el crecimiento económico, la parte positiva es aquella que contempla al primero como elemento fundamental para la consecución de un mejor desempeño de la actividad productiva. No obstante, los autores también resumen que, pese al avance alcanzado, aún no existe una conclusión única sobre el vínculo que puede existir entre ambas variables. En términos generales, se conoce que el sistema financiero sirve como mecanismo para canalizar el ahorro al sistema productivo de la economía, situación que implicaría que el PIB debe causar (en el sentido de Granger) al crédito. Por otro lado, es aceptado que cuando el dinero es endógeno, la causalidad va del crédito a la inversión.

En lo correspondiente al análisis empírico es extensa la gama de estudios que tratan de probar una causalidad entre las variables en estudio. Aunque existen muchas diferencias en torno de la dirección de dicha causalidad y los métodos econométricos utilizados, sí hay un consenso general de que existe relación entre el sistema financiero y el crecimiento económico. Los estudios seminales desarrollados por Goldsmith (1969) indican la existencia de una robusta relación de causalidad positiva entre 
la evolución del sistema financiero y los cambios en las tasas de crecimiento económico. Dentro de esta línea McKinnon (1973 en referencias) y Shaw (en referencias 1973) obtuvieron resultados similares. Por tanto, y de acuerdo con estos autores, se infiere que el desarrollo del sistema financiero es favorable para impulsar la actividad productiva de los países. Arestis, Luintel y Luintel (2005) analizaron la evidencia estadística para el caso de países en desarrollo; encontraron que existe una causalidad que va de la estructura financiera hacia el PIB per cápita real y al capital per cápita. Como ya se mencionó, King y Levine (1993) encontraron resultados similares para una muestra amplia de ochenta países.

Para el caso de México, De la Cruz y Núñez (2011) plantean que existe una correlación positiva entre la actividad industrial y el comportamiento de la Bolsa Mexicana de Valores (BMV), medido por el índice de precios y cotizaciones (IPC). Los autores plantean, en una primera etapa, que la inflación tiene una relación positiva con su volatilidad; a partir de lo cual comprueban la presencia de una relación negativa entre dicha variabilidad y los rendimientos reales de la BMV. En este sentido, la investigación concluye que el IPC y la actividad industrial están cointegradas y exhiben una causalidad bidireccional. La implicación de los resultados sostiene que el banco central puede intervenir en el crecimiento industrial vía el control de la inflación.

Por otro lado, Rodríguez y López (2009) concluyen que, para el caso mexicano, se encuentra una relación de causalidad bidireccional entre el desarrollo del sistema financiero y el crecimiento económico. Así mismo, Tinoco, Torres y Venegas (2009) consideran que la dirección causal entre las dos variables en estudio es unidireccional del sistema financiero al crecimiento; y que la represión financiera limita las tasas de crecimiento de la actividad económica.

Por su parte Cotler y Rodríguez (2010) determinan que en el caso mexicano no se puede rechazar la posibilidad de que el financiamiento tenga un impacto en el bienestar. Al respecto, Friedman (1957) planteó que las formas en que los agentes eligen su consumo óptimo dependen de una medida a largo plazo del ingreso, en términos conceptuales, del ingreso permanente. La implicación económica más importante de dicha teoría es que el crédito constituye una forma de suavizar el consumo en el tiempo ante un patrón de ingresos variables (Martínez, 1997). Cuando tal 
hipótesis se rechaza, lo más probable es que exista una situación en la que estén presentes imperfecciones en el mercado de crédito y restricciones de liquidez; por lo que el consumo privado dependería, más bien, del ingreso disponible. Hall (1978) plantea la hipótesis del ingreso permanente (HIP) de Friedman (1957) como un problema de maximizar una función de utilidad que depende del consumo, sujeta a una restricción presupuestaria donde la riqueza del agente iguala sus ingresos en el tiempo (Y). El autor propone una regresión para medir la capacidad de los agentes de planificar su consumo (C) intertemporal:

$$
C_{t}=\alpha_{\mathrm{o}}+\alpha_{1} C_{t-1}+\lambda Y_{t}-\lambda Y_{t-1}+\varepsilon_{t}
$$

En su estudio para México, Ponce (2003) concluye que en el periodo 1980-200o el parámetro $\lambda$ alcanzó un valor de 0.452. Lo que significa que no se cumple la HIP, es decir, parte del consumo en el tiempo $t$ se encuentra restringido por el ingreso variable o disponible. Las implicaciones del resultado no son menores; el hecho de que la HIP no se cumpla supone que el poder de ahorro de los agentes es pequeño, lo que impacta al crecimiento de forma negativa. En términos generales, el consumo en México presenta restricciones de liquidez. En el mismo estudio, el autor considera que después de la crisis de 1995 el parámetro $\lambda$ ascendió sólo para ese periodo a 0.97, esto es, por cada punto porcentual que aumenta el ingreso corriente, casi todo se destina al consumo. Más aún, al elaborar una regresión donde se incluye al crédito como regresor se obtiene un resultado de significancia estadística, lo que fortalece la idea de que una mejora en el mercado de crédito puede incentivar el consumo privado.

\section{UNA RESEÑA DEL CASO MEXICANO}

Dos tendencias claras distinguen el proceso de crecimiento del crédito en México. Una se presenta en la década de los ochenta, y se caracterizó por la presencia de variadas reformas estructurales cuyo objetivo principal consistió en liberalizar el mercado. La problemática década perdida impidió que durante los primeros años de la misma existiera un financiamiento de la banca nacionalizada hacia las empresas privadas y al 
consumo. Fue hasta el periodo 1988-1994 cuando el crédito otorgado por la banca comercial representó la principal fuente de financiamiento de las empresas (Garay, 2000). De acuerdo con el autor, antes de la crisis de 1995 el crédito al sector privado tuvo una tasa de crecimiento real de 39\% (en términos nominales fue de casi 200 por ciento).

Por otro lado, después de la crisis de 1994 hubo una considerable reducción en los volúmenes de crédito otorgado por la banca comercial a la economía, de tal manera que, hasta la fecha, aún no se alcanzan los niveles de financiamiento que se tenían antes de 1995. En la actualidad, más de la mitad del crédito al sector privado de la economía proviene de fuentes alternativas de financiamiento (Mantey de Anguiano, 2007), algo que limita la capacidad del sistema financiero para impulsar el crecimiento económico.

Las razones de este quiebre en la tendencia del crédito son de distinta naturaleza. En relación con la parte microeconómica, se tiene que la función de los bancos para captar el ahorro y distribuirlo a las actividades económicas del país había sido determinante hasta principios de la década de los setenta. Sin embargo, a partir de entonces la instrumentación de los cajones selectivos obligó a los intermediarios financieros a destinar un monto de crédito al gobierno, que, de manera creciente, lo utilizó para cubrir su déficit. En consecuencia, el sector público absorbió gran parte del volumen de financiamiento en la economía, lo que provocó una caída en la penetración financiera al disminuir el monto de recursos disponibles para el sector privado (Millán, 1999). Debido al aumento del déficit público, para principios de la década de los ochenta se tomaron medidas de desregulación financiera, que consistieron en la desaparición de los cajones selectivos y en la adopción de un sistema libre de tasas de interés. Aunado a lo anterior, la privatización de la banca terminó por impulsar el volumen del crédito, con lo cual los bancos podían prestar prácticamente sin restricciones a quien quisieran, con un sistema de libre tasa de interés. No obstante, el inapropiado mecanismo de privatización, la poca experiencia de las casas de bolsa beneficiadas por la adquisición de los bancos, la inadecuada regulación y supervisión financiera, el excesivo crédito al consumo, la precaria valuación del riesgo y el rezago tecnológico del sector, provocaron un aumento de los empréstitos por encima de la dinámica real de la economía. A raíz de estos desequilibrios y de la profunda crisis económica, los resultados para el sistema de pagos del país fueron 
devastadores. El PIB cayó 6.2\% durante 1995, retroceso del cual tomó un par de años recuperarse. En tanto que, en el caso del crédito otorgado por la banca comercial, para finales de la década de los noventa había decaído 46.4\% respecto de su nivel previo a la recesión. Por otra parte, el rescate bancario derivado de la crisis ha representado un gran pasivo para las finanzas públicas; baste recordar que al cierre de 2010 se acumularon requerimientos financieros por más de 773.6 mil millones de pesos.

La desregulación financiera y la extranjerización de la banca, sin duda, cambiaron la estructura financiera del país; no obstante, ha traído efectos negativos en términos del financiamiento a la producción y a la estabilidad financiera (Levy, 2007). En general, el nuevo sistema no ha logrado diversificar la colocación de bonos, por lo que se mantiene así la dominación de los bonos públicos, cuya emisión correspondió a $75.5 \%$ de la deuda interna en 2010. En este sentido, el sector productivo es el más afectado por la escasez de recursos, lo que, aunado a una época de alta especulación, ha disociado al sistema financiero del crecimiento económico (Levy, 2007). Por tanto, no debe sorprender que, si bien los efectos de la crisis financiera de 1995 se diluyeron relativamente rápido, el financiamiento al sector privado siguió viviendo un periodo de franca desaceleración. Durante el periodo 1995-1999 dicho tipo de crédito disminuyó 53.3\% en términos reales (Gráfica 1).

\section{Gráfica 1}

\section{Crédito al sector industrial y al consumo como proporción del crédito total}

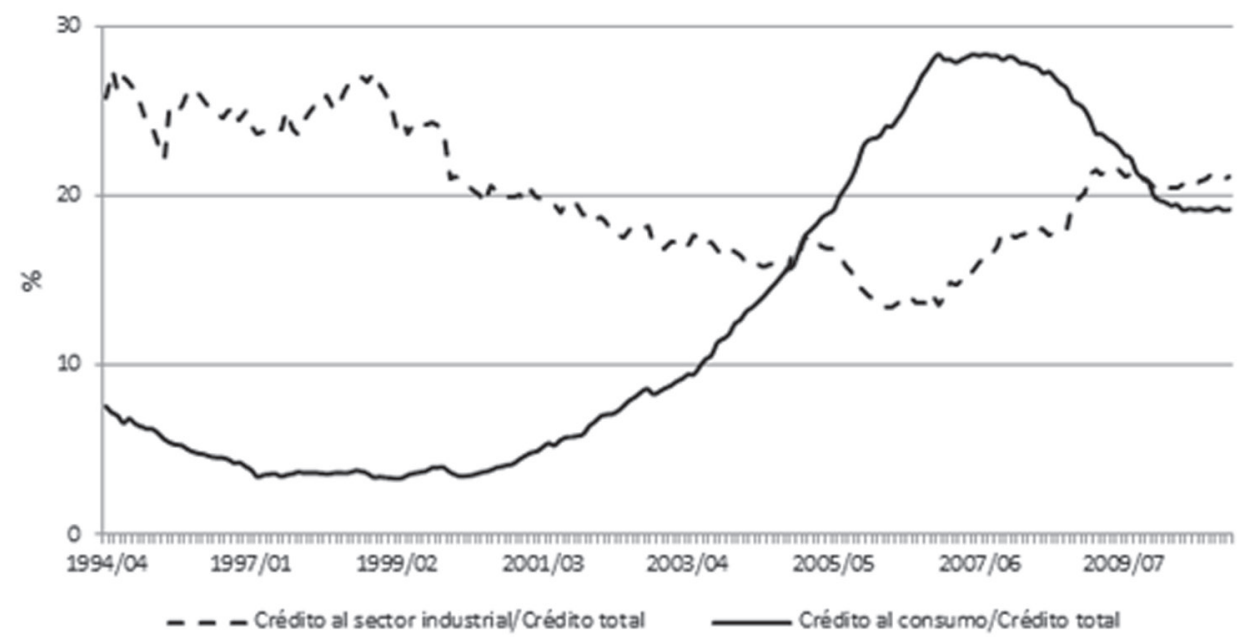

Fuente: elaboración propia con información de la Comisión Nacional Bancaria y de Valores y el INEGI 
Aunado a lo anterior, si se mide la trayectoria del porcentaje del crédito como proporción del PIB, se puede apreciar una caída significativa. Además, la conformación de un oligopolio bancario que privilegia los altos márgenes de utilidad ha provocado un escaso crédito al sector productivo, de manera particular al destinado tanto a las pequeñas y medianas empresas como a los procesos de innovación científica y tecnológica. En México, los cuatro bancos más importantes detentan 70\% del mercado (CNBV, 2011); asimismo, las ganancias de dichos bancos representaron en 2010 10\% de las ganancias globales. Su posición dominante en el mercado les ha permitido elevar considerablemente la tasa de interés activa a lo largo del tiempo, lo que provoca una enorme brecha entre la tasa de depósitos y la tasa libre de riesgo, es decir, el margen financiero libre de riesgo (Palacios, 2009).

Sin duda, la crisis de 1995 fue un parteaguas en el mercado crediticio mexicano. Aun con la entrada de la banca extranjera no se han podido alcanzar los niveles de crédito que se tenían antes de la recesión. El costo que ha pagado el mercado crediticio se traduce en un debilitamiento sistemático que solamente fue interrumpido hasta el año 2003, cuando se inició una incipiente recuperación. Sin embargo, la crisis financiera de 2008 volvió a frenar los flujos de capital hacia el sistema productivo. En este punto es importante resaltar que el impacto negativo que en el mercado del crédito enfrentaron otros países de Latinoamérica, como Chile y Brasil $^{1}$, fue mucho menor que el acontecido en México, situación que les permite mantener un ritmo de crecimiento económico superior. Lo anterior se debe a que, en este último, la presencia de la banca extranjera es dominante, y como la reciente crisis financiera tuvo su origen en naciones que, a su vez, son las matrices de los bancos en México, el sistema bancario quedó a expensas de la volatilidad global que se enfrentó entre 2008 y 2009, lo cual se tradujo en una reducción del crédito local.

\footnotetext{
${ }^{1}$ En este sentido, la cartera de crédito total en Brasil aumentó 20.5\% en 2010.
} 


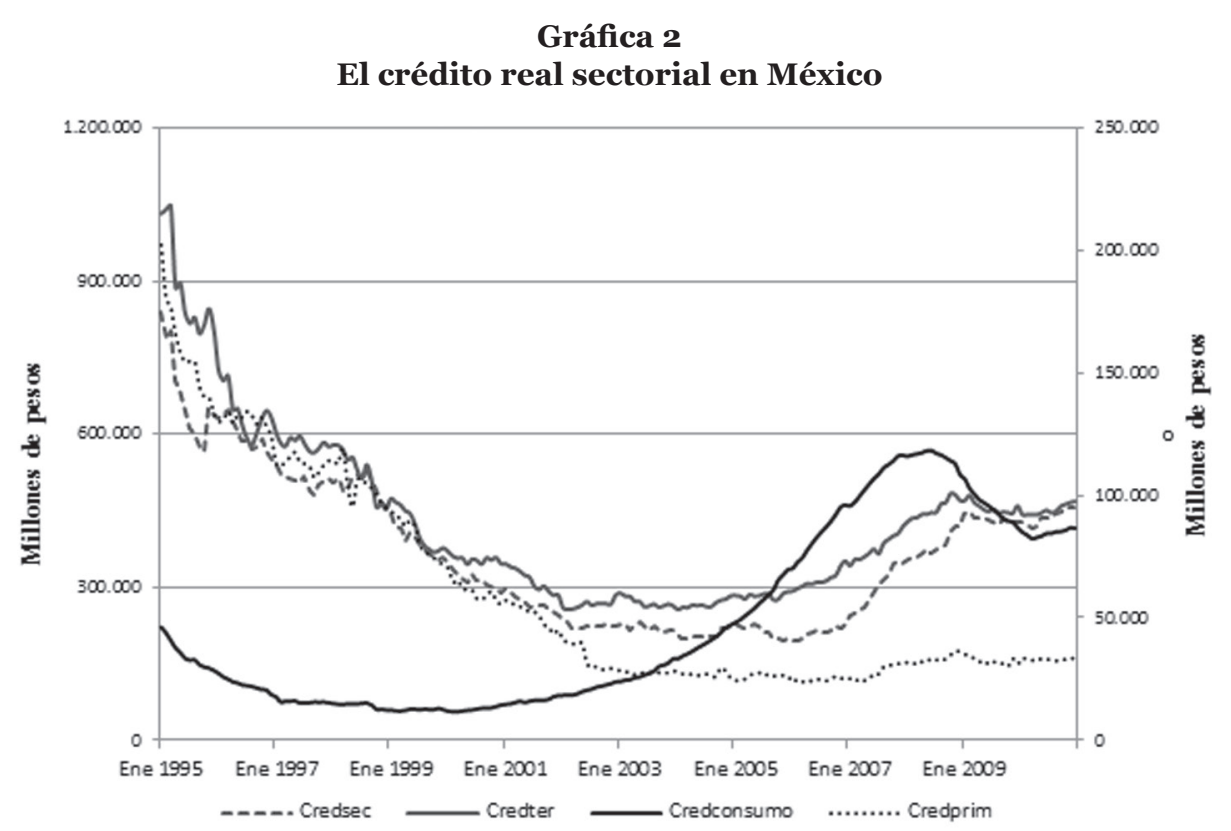

Fuente: elaboración propia con información de la Comisión Nacional Bancaria y de Valores y el INEGI

Como se ha mencionado, existe una amplia literatura cuya hipótesis establece una relación de causalidad que va del ahorro al crecimiento, que crea un mecanismo que acelera la dinámica económica. En este sentido Garrido y Prior (2010) plantean la hipótesis de que una forma de incentivar el mercado crediticio en México, y en general su sistema financiero, es a partir de la creación de un círculo virtuoso que empiece con un proceso robusto de bancarización. Lo descrito implica incentivar el ahorro interno y el acceso al crédito a los diversos sectores productivos. No obstante lo anterior, tampoco puede dejarse de lado la evidencia empírica que señala al crecimiento como motor del ahorro (Saltz, 1999). Los modelos neoclásicos generalmente proponen que tasas de ahorro mayores generan mayores incrementos de crecimiento de la economía. Los resultados de Saltz (basados en las pruebas de causalidad de Granger para modelos de corrección de error) muestran que no se encontró causalidad en ninguna dirección para Colombia, Jamaica, Perú y Filipinas. En el caso de Argentina y Taiwan la relación es unidireccional de la tasa de ahorro hacia el 
PIB, para México y República Dominicana la relación es bidireccional. Las conclusiones de Saltz indican que existe un vínculo diferenciado entre el ahorro y el crecimiento, de acuerdo con el país que se analice, por lo que el objetivo del presente artículo es identificar el tipo de relación que guardan los distintos tipos de crédito del sector bancario en relación con la actividad productiva de México.

En el contexto actual, y al tratar de dar una respuesta al cuestionamiento anteriormente planteado, surge una pregunta necesaria: ¿puede ser contradictorio un aumento desproporcionado del crédito en la economía? De acuerdo con Avendaño (2010), una de las causas por las que el sistema financiero mexicano sucumbió en 1995 fue la expansión desmedida del crédito al sector privado. A nivel global, las crisis de los años noventa fueron precedidas por una etapa de desregulación excesiva de los flujos financieros, una expansión de la deuda soberana y privada, así como por elevados déficits en la balanza de pagos. En consecuencia, ha existido una mayor preocupación por mantener en estricta revisión el mercado de crédito, y con ello evitar los efectos adversos que sufre la economía después de una inusual expansión crediticia, es decir, de aquella que sobrepasa la tendencia de largo plazo del crédito privado (Mendoza, 2008).

Lo anterior podría describir lo que se conoce como un boom de crédito, fenómeno que generalmente está asociado con periodos de expansión económica, incrementos de los flujos de capital, aumento en los precios de bienes raíces y déficits externos. Una característica de dicho fenómeno en los mercados emergentes es que variables macroeconómicas como la razón crédito/PIB, la depreciación y los déficits suelen ser mayores y recurrentes, lo cual provoca desequilibrios prolongados (Mendoza, 2008). Aunque no todos los booms de crédito culminan en una crisis, las más recientes han estado asociadas al fenómeno en cuestión. Mendoza señala que aumentos en la razón crédito/PIB están asociados con episodios de crisis gemelas, mismas que pueden ser devastadoras en cualquier sistema financiero y, por ende, para el bienestar de la población. Por esta razón, un primer paso a realizar es estimar si en los últimos años se ha presentado un boom de crédito en México, con el fin de establecer si fue antecedente de la reciente recesión económica. Lo anterior no es trivial, ya que en 2008 el sector público instrumentó reformas para crear organismos autorreguladores de las instituciones de crédito, otorgó, además, mayores 
facultades a la CNBV para vigilar la evolución del crédito, y adoptó una regulación encaminada a fortalecer las reglas del capital (Avendaño, 2010). En conjunto, todo ello apunta a la necesidad que se tenía para prevenir la generación y explosión de un boom de crédito.

\section{METODOLOGÍA Y DATOS}

Para la consecución del objetivo planteado en primera instancia, y de acuerdo con Mendoza (2008), se estima el boom de crédito con el fin de establecer si previo a la crisis de 2009 existían elementos que señalaran al otorgamiento de préstamos como algo no sustentable por el crecimiento económico de México. Como segundo paso, se analizan los vínculos causales entre la dinámica de la economía y el crédito. La información utilizada corresponde a los componentes sectoriales del Índice Global de Actividad Económica (IGAE), ${ }^{2}$ así como del crédito bancario otorgado a los mismos. Por definición, el IGAE mide la evolución de la actividad económica del país y toma la base de la contabilidad nacional para su elaboración, por lo que es un buen estimador de la tendencia de la actividad económica en el corto plazo. Por otro lado, las series del crédito consideradas en el análisis se refieren al monto total y desagregado del financiamiento otorgado por la banca comercial a los diferentes sectores económicos del país. La idea de utilizar dicha variable es para medir el impacto que tiene el sistema financiero en el crecimiento de la economía mexicana. La frecuencia de la información es mensual y el horizonte temporal va de enero de 1995 a noviembre de 2010. Las series de crédito fueron deflactadas con el Índice Nacional de Precios al Consumidor (INPC). El IGAE y el INPC se tomaron del Instituto Nacional de Estadística, Geografía e Informática, INEGI, y el crédito de la base estadística del Banco de México.

En el análisis de causalidad se parte de la hipótesis nula de que "Y no causa X, dadas otras variables" y es probada mediante el estadístico $F$ estándar definido por Ghartey (1993). Sin embargo, y con el fin de que las pruebas de causalidad sean estadísticamente significativas, en una primera

${ }^{2}$ Para el cálculo del IGAE se utiliza la misma metodología con la que se calcula el PIB trimestral. Se considera que este indicador es una buena aproximación del producto interno bruto. 
etapa es fundamental determinar si las series son estacionarias y, en dado caso de que no sea así, determinar si existen relaciones de largo plazo entre las mismas, es decir, estimar si hay relaciones de cointegración entre ellas. Dado que en las series económicas mexicanas se pueden manifestar cambios estructurales, fruto de los diversos problemas que en la última década se han presentado, es necesario asegurarse de que el orden de integración es determinado de manera adecuada, para ello se realizan dos pruebas de raíz unitaria. Primero, se aplica tanto la prueba de Dickey-Fuller Aumentada (ADF) como la de Kwiatkowski, Phillips, Schmidt y Shin (KPSS). En este sentido, se sigue el proceso de decisión propuesto por Charemza y Deadman (1992) para encontrar el modelo estadísticamente pertinente. Lo descrito es relevante ya que los trabajos de Toda y Phillips (1993a) muestran que los modelos autorregresivos no son confiables cuando se estiman relaciones causales en series que no son estacionarias. Algo similar ha sido establecido por Sims, Stock y Watson (1990), quienes señalan que las distribuciones asintóticas no deben ser utilizadas para probar las restricciones en un modelo VAR cuando las variables no son estacionarias. Además, si en un VAR existe un grupo de variables con tendencias estocásticas comunes, se afirma que están cointegradas, y, entonces, puede estimarse un VEC o un VAR, este último debe seguir la propuesta de Toda y Yamamoto (1995). No obstante, Engle y Yoo (1987), Lin y Tsay (1996) y Naka y Tufte (1997) señalan las ventajas de aplicar un VEC. Básicamente, los autores muestran que en el largo plazo los resultados del modelo restringido tienen un mejor ajuste. Conforme estas recomendaciones, en el presente estudio se aplica un VEC para los casos en donde las variables no estacionarias se encuentren cointegradas. La elaboración de un VEC parte de la determinación óptima del número de rezagos a incluir en el modelo, razón por la cual se utilizan los criterios de información. En consecuencia, se construyeron los VAR correspondientes y se determinó el número de rezagos óptimo en función de los criterios de Akaike, Schwarz y Hannan-Quinn. Para determinar la posibilidad de que existan relaciones de largo plazo entre las variables se aplicó la prueba de Johansen (1990) . En el caso de que las series no estén cointegradas se elabora un VAR en diferencias para determinar la causalidad sin tener problemas de relaciones espurias. 
En términos matemáticos la elaboración de un VAR se realiza a través de un proceso autorregresivo multivariado del tipo:

$$
\mathbf{y}_{t}=\boldsymbol{\mu}+\mathbf{A}_{1} \mathbf{y}_{t-1}+\ldots \mathbf{A}_{p} \mathbf{y}_{t-p}+\boldsymbol{\varepsilon}_{t}
$$

En donde $\mathbf{y}_{t}$ es un vector $n x 1$ de variables. Si las variables son integradas de orden 1[I (1)] y están cointegradas, el proceso autorregresivo anterior puede ser representado como un VEC de la siguiente manera:

$$
\Delta \mathbf{y}_{t}=\boldsymbol{\mu}+\boldsymbol{\Pi} \mathbf{y}_{t-1}+\sum_{i=1}^{p-1} \boldsymbol{\Gamma} \Delta \mathbf{y}_{t-1}+\boldsymbol{\varepsilon}_{t}
$$

Donde

$$
\boldsymbol{\Pi}=\sum_{i=1}^{p} \mathbf{A}_{\mathrm{i}}-\mathbf{I} \mathbf{y} \boldsymbol{\Gamma}_{t}=-\sum_{j=i+1}^{p} \mathbf{A}_{j}
$$

El rango de $\boldsymbol{\Pi}$ determinará el número de vectores de cointegración linealmente independientes que pueden existir entre los componentes de $\mathbf{y}_{t}$.

Sea $r$ el rango de la matriz $\Pi$ y $k$ su número de columnas. Si $\Pi$ es de rango reducido, es decir, $r<k$, entonces decimos que existen dos matrices que tampoco son de rango completo $\boldsymbol{\alpha}$ y $\boldsymbol{\beta}$, tal que $\Pi=\boldsymbol{\alpha} \boldsymbol{\beta}$ y $\boldsymbol{\beta} \mathbf{y}_{\mathrm{t}-1}$ es I (o), en donde $\boldsymbol{\beta}^{\prime}$ representa los vectores de cointegración y $\boldsymbol{\alpha}$ los parámetros de ajuste del desequilibrio en el proceso. Por lo tanto, el problema se reduce a encontrar $r$. La causalidad se determina al aplicar una prueba $F$ sobre los rezagos de las variables bajo estudio.

\section{RESULTADOS}

En primer lugar, se realizó la estimación de la tendencia del crédito al consumo para establecer un posible boom de crédito antes de la crisis de 2009. Como se aprecia en la gráfica 3, hacia fines de 2007 ya existía un aumento del crédito por encima de su tendencia. Lo anterior supone que, previo a la recesión, la evolución de la variable ya se encontraba por encima de las capacidades reales de la economía, hecho que, por sí solo, anunciaba que en el mediano y largo plazos era complicado mantener el 
vigor de su desempeño. La relevancia de lo descrito radica en que, si el crecimiento económico depende del crédito otorgado al consumo, ello podría implicar que al limitarse el otorgamiento del mismo, a la par se condiciona negativamente al desempeño de la economía mexicana. Para establecer la posibilidad citada es necesario realizar las pruebas de causalidad ya descritas.

Gráfica 3

Crédito al consumo (serie original y de tendencia)

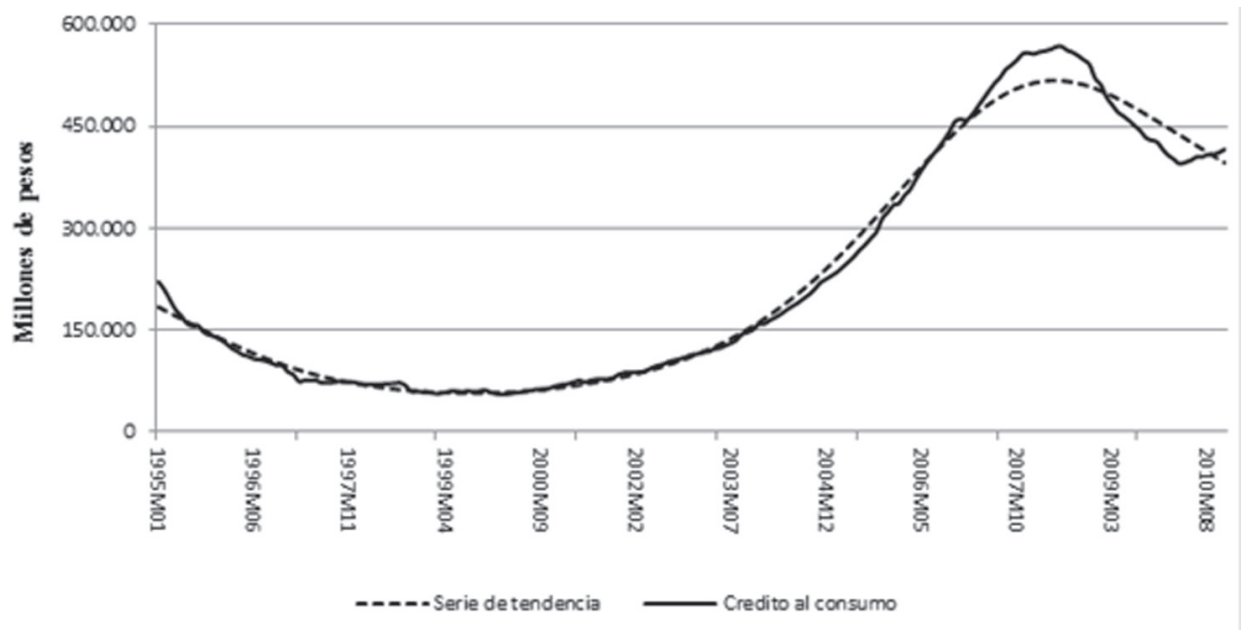

Fuente: elaboración propia con información de la Comisión Nacional Bancaria y de Valores y el INEGI.

En correspondencia, se determinó el orden de integración de las series bajo estudio, para lo cual se emplearon las pruebas de raíz unitaria Dickey-Fuller aumentada (ADF) y Kwiatkowski, Phillips, Schmidt y Shin (KPSS). Los resultados muestran que todas las series son integradas de orden 1 [I (1)]. 


\section{Cuadro 1a}

Análisis de raíz unitaria (ADF)

\begin{tabular}{|l|c|c|c|c|c|}
\hline \multicolumn{1}{|c|}{ Serie $(\mathrm{ln})$} & $\begin{array}{c}\text { Estadistico } t \\
(\text { en niveles) }\end{array}$ & $\begin{array}{c}\text { p-value } \\
(\text { en niveles })\end{array}$ & $\begin{array}{c}\text { Estadistico } t \\
\left(\Delta \boldsymbol{y}_{t}\right)\end{array}$ & $\begin{array}{c}p \text {-value } \\
\left(\Delta \boldsymbol{y}_{t}\right)\end{array}$ & $\mathrm{I}(\mathrm{d})$ \\
\hline IGAE & -2.850591 & 0.1814 & -6.252711 & 0.0000 & $\mathrm{I}(1)$ \\
\hline IGAESEC & -2.570579 & 0.1010 & -9.399404 & 0.0000 & $\mathrm{I}(1)$ \\
\hline IGAEPRIM & -2.682543 & 0.2452 & -14.81696 & 0.0000 & $\mathrm{I}(1)$ \\
\hline IGAETER & -1.709968 & 0.7433 & -14.01843 & 0.0000 & $\mathrm{I}(1)$ \\
\hline CREDITO & -1.793855 & 0.7041 & -12.26806 & 0.0000 & $\mathrm{I}(1)$ \\
\hline CREDPRIM & -3.037641 & $0.0333 *$ & -14.63736 & 0.0000 & $\mathrm{I}(1)$ \\
\hline CREDSEC & -0.578355 & 0.9789 & -12.76226 & 0.0000 & $\mathrm{I}(1)$ \\
\hline CREDTER & -1.709968 & 0.7433 & -14.01843 & 0.0000 & $\mathrm{I}(1)$ \\
\hline CREDCONSUMO & -2.242457 & 0.4629 & -3.465147 & 0.0462 & $\mathrm{I}(1)$ \\
\hline
\end{tabular}

Nota: *No se puede rechazar la hipótesis nula a $1 \%$.

\section{Cuadro 1b}

\section{Análisis de raíz unitaria (KPSS) ${ }^{3}$}

\begin{tabular}{|l|r|r|r|r|r|}
\hline \multicolumn{1}{|c|}{ Serie $(\boldsymbol{l n})$} & $\begin{array}{c}\text { Estadistico } \\
\text { (en niveles) }\end{array}$ & $\begin{array}{c}\text { Valor } \\
\text { critico 5\% } \\
\text { (en niveles) }\end{array}$ & $\begin{array}{c}\text { Estadistico } \\
\left(\Delta \boldsymbol{y}_{\boldsymbol{t}}\right)\end{array}$ & $\begin{array}{c}\text { Valor } \\
\text { crítico5\% } \\
\left(\Delta \boldsymbol{y}_{\boldsymbol{t}}\right)\end{array}$ & $\mathrm{I}(\mathrm{d})$ \\
\hline IGAE & 0.235760 & 0.146000 & 0.080297 & 0.146000 & $\mathrm{I}(1)$ \\
\hline IGAESEC & 0.249664 & 0.146000 & 0.082674 & 0.146000 & $\mathrm{I}(1)$ \\
\hline IGAEPRIM & 0.166534 & 0.146000 & 0.243324 & 0.463000 & $\mathrm{I}(1)$ \\
\hline IGAETER & 0.139784 & 0.146000 & & & $\mathrm{I}(0)$ \\
\hline CREDITO & 0.422520 & 0.146000 & 0.120663 & 0.146000 & $\mathrm{I}(1)$ \\
\hline CREDPRIM & 0.386006 & 0.146000 & 0.085344 & 0.146000 & $\mathrm{I}(1)$ \\
\hline CREDSEC & 0.405246 & 0.146000 & 0.079339 & 0.146000 & $\mathrm{I}(1)$ \\
\hline CREDTER & 0.422628 & 0.146000 & 0.094257 & 0.146000 & $\mathrm{I}(1)$ \\
\hline CREDCONSUMO & 0.298290 & 0.146000 & 0.399943 & 0.146000 & $\mathrm{I}(2)$ \\
\hline
\end{tabular}

\footnotetext{
${ }^{3}$ En el caso de las series del IGAE para el sector terciario y el crédito al consumo tomamos el resultado de la prueba $\mathrm{ADF}$.
} 
Dado que las series no son estacionarias es necesario establecer la posibilidad de que se encuentren cointegradas, razón por la cual se procedió a la aplicación de la prueba de Johansen. En el cuadro 2 de los anexos se presentan las variables para las que se encontró la existencia de una relación de largo plazo, en cuyo caso se elaboró un VEC. El resto de las estimaciones fue mediante un VAR en diferencias. Los vectores autorregresivos y de corrección de error se elaboraron para cada par de series y se determinaron a partir de los rezagos óptimos seleccionados por los criterios de información de Akaike, Schwarz y Hannan-Quinn. Una vez que dichos lags se establecieron, en el caso de que las variables se encontraran cointegradas, se aplicó un VEC con los rezagos que habían sido establecidos para el VAR correspondiente.

Los resultados estadísticos establecen la existencia de una relación de cointegración entre el IGAE y el crédito total. De igual forma, se puede determinar dicho vínculo entre el financiamiento al consumo y el IGAE, así como entre el IGAE terciario y el crédito que se otorga a ese sector. A partir de lo anterior se aplican las pruebas de causalidad y se establece la dirección de cada relación. Los resultados obtenidos se presentan en el cuadro 2. Las pruebas de causalidad permiten afirmar que existe una relación causal del financiamiento total otorgado a la economía sobre el IGAE. Con dicho resultado se puede plantear que, de manera general, la expansión del crédito genera crecimiento, algo que se encuentra en concordancia con la teoría que postula al sistema financiero como factor de desarrollo económico. No obstante, las estimaciones sectoriales realizadas hacen posible identificar los canales de transmisión de los efectos entre el sector bancario y la economía real.

La estimación realizada establece la presencia de una interacción causal bidireccional entre el crédito privado otorgado al consumo y el IGAE, es decir, se tiene un círculo positivo que vincula al financiamiento con respecto al desempeño del sector real, y que el vínculo es de largo plazo. Como ya se describió, esta dinámica se encuentra fundamentada en el importante incremento que el crédito al consumo ha mostrado desde finales de la década de los años noventa.

Lo anterior se refuerza con la relación de causalidad bidireccional que prevalece entre el IGAE terciario y financiamiento al consumo (ver apéndice 4). De igual manera la interrelación causal, en este caso unidireccional, 
que va del el crédito al sector terciario y el IGAE hace posible inferir que los recursos canalizados por el sistema bancario hacia el consumo y los servicios tienen un impacto positivo sobre la economía sectorial y total de México.

\section{Cuadro 2}

Causalidad en el sentido de Granger

\begin{tabular}{|lcccccc|}
\hline Variable dependiente & Crédito & Credconsumo & Credprim & Credsec & Credter & Causalidad \\
\hline IGAE & 0.0221 & 0.028 & 0.8792 & 0.2159 & 0.319 & Crédito $\rightarrow$ IGAE \\
& (VEC) & (VEC) & $\ldots . .$. & & Credconsumo $\rightarrow$ IGAE \\
\hline
\end{tabular}

\begin{tabular}{|l|l|l|}
\hline Variable dependiente & IGAE & Causalidad \\
\hline Crédito & $0.0653($ VEC $)$ & No hay causalidad \\
Credconsumo & $0.0087($ VEC) & IGAE $\rightarrow$ Credconsumo \\
Credprim & 0.1531 & No hay causalidad \\
Credsec & 0.1603 & No hay causalidad \\
Credter & $0.0479($ VEC $)$ & Credter $\rightarrow$ IGAE \\
\hline
\end{tabular}

No obstante, la modelación elaborada no generó resultados estadísticamente significativos que vincularan los distintos tipos de crédito con el IGAE total o sectorial (ver apéndice 3). La relevancia de lo anterior es que ello delimitaría el impacto que el sistema bancario en México tiene sobre la actividad económica. Cuando el crédito no tiene una relación con el sector primario y secundario, se tiene merma en la capacidad productiva del país en dos sectores estratégicos. En el primer caso, se tiene en los que están directamente involucrados con la alimentación y la extracción de recursos naturales que inciden tanto en el bienestar de la población como en la generación de insumos básicos para la producción. En lo que respecta al sector secundario, la ausencia de una relación con el crédito restringe el desarrollo de las capacidades de innovación y tecnología que 
están asociadas a sectores industriales como el de manufacturas, de la construcción y el eléctrico. En conjunto, la falta de penetración del financiamiento hacia actividades económicas que no son de servicios provoca una disminución en la competitividad y la generación de valor agregado. Dicha situación se ha exacerbado por la caída del crédito encauzado hacia los sectores primario y secundario, algo que ocurrió después de la crisis de 1995 .

Una implicación adicional es que la merma en el financiamiento hacia actividades industriales que generan mayor valor agregado provoca un menor crecimiento en el producto interno bruto (PIB) potencial, lo cual, a su vez, puede incidir negativamente sobre los efectos que el crédito al consumo produce en la economía. La implicación radica en que, ante un modesto crecimiento, el excesivo aumento de recursos canalizados hacia el consumo y los servicios generan auges crediticios, es decir, préstamos que no se encuentran sustentados por la dinámica real de la economía, que en el mediano y largo plazos terminan con contracciones en la tasa de crecimiento.

\section{CONCLUSIONES}

Los resultados encontrados plantean tres hechos relevantes. En primer lugar, que el único tipo de crédito sectorial que impacta a la economía es el otorgado al consumo y los servicios. Si bien el estudio muestra que, en términos generales, no se puede rechazar la hipótesis de que el crédito total otorgado por la banca comercial causa un aumento en la actividad productiva de México, es importante matizar que la relación positiva y de largo plazo, que en realidad guarda el crecimiento con el crédito, tiene su verdadero vínculo con los recursos encaminados al consumo. Al encontrarse que existe una relación de causalidad bidireccional entre el IGAE y dicha variable, se puede concluir que prevalece una estrecha interacción a través del impulso al ahorro y de los procesos de bancarización que incentivan la demanda agregada. En segunda instancia, se encontró que la evolución del financiamiento al consumo provocó un boom crediticio previo a la crisis de 2009. Dado que aumentos desmedidos del financiamiento traen consecuencias negativas en la economía, mediante crisis bancarias y 
financieras, es relevante considerar que debe realizarse una adecuada regulación del sistema bancario, con la finalidad de que se eviten crisis similares a las observadas en los años noventa. Finalmente, el estudio muestra que existe una interacción limitada entre el sector real y el sistema bancario, lo anterior tiene dos elementos: 1) los recursos canalizados a los sectores productivos han mostrado una evolución más moderada respecto de lo observado en el caso del consumo, y 2) el desempeño económico de dichos sectores ha sido modesto. Este resultado tiene una connotación relevante: si se desea aumentar las capacidades productivas y competitivas de México será imprescindible que se solvente la limitante citada, ya que la ausencia de vínculos entre los créditos otorgados y el crecimiento económico indica que parte de la esfera financiera no tiene relación con la generación de valor agregado del país.

Por tanto, debe plantearse que si bien se han encontrado elementos de interacción positiva entre el sistema bancario y la economía real, no puede dejarse de lado que los mismos aún son incipientes y heterogéneos, cuestión que presenta un desafío que la política económica y las instituciones financieras deben resolver para aumentar las capacidades productivas de México. 


\section{REFERENCIAS}

Arestis, P., A. Luintel y K. Luintel. 2005. Financial structure and economic growth, Cambridge University, CEPP working paper, núm. 06/05.

Avendaño, O. 2010. El sistema financiero internacional. Instituciones y crisis financieras, Porrúa- ITESM CCM, México.

Bencivenga, V. y B. Smith. 1991. Financial intermediation and endogenous growth, The Review of Economic Studies, (58): 195-209.

Charemza, W. y D.F. Deadman. 1992. New Directions in econometric practice: General to specific modeling, cointegration, and vector autoregression, Aldershot: Edward Elgar Publishing.

Cotler, P. y E. Rodríguez. 2010. "Microfinanzas y la tenencia de activos no financieros en México", Investigación Económica, LXIX(274): 63-86.

De la Cruz, J.L. y J.A. Núñez. 2011. "Causalidad entre la Bolsa Mexicana de Valores y la actividad económica del sector real”, Eseconomía. 6(30).

Engle, R. y B. Yoo. 1987. Forecasting and testing in co-integrated systems, Journal of Econometrics, 35: 143-159.

Friedman, M. 1957. A theory of consumption function, Princeton University Press

Garay, A. 200o. Evolución del crédito en México, en A. Núñez y R. Martínez, Las finanzas del sistema federal mexicano, INAP/Cámara de Diputados, México, pp. 413-466. 
Garrido, C. y F. Prior. 2007. Bancarización y microfinanzas. Sistemas financieros para las Mypymes como un dilema central para el desarrollo económico en México, en J.L. Calva (comp.) Financiamiento del crecimiento económico, UNAM/Porrúa/Cámara de Diputados, México, pp. 57-77.

Ghartey, E. 1993. Causal relationship between exports and economic growth: Some empirical evidence in Taiwan, Japan and the U.S., Applied Economics, 25: 1145-1152.

Goldsmith, W. 1969. Financial structure and development, New Heaven, Yale University Press.

Hall, R. 1978. Stochastic implications of the life cycle- permanent income hypothesis: Theory and evidence, Journal of Political Economy, 86: $971-987$.

King, R. y R. Levine. 1993. Finance and growth: Schumpeter might be right, The Quarterly Journal of Economics, 108(103): 717-737.

Levy, N. 2007. Financiamiento del crecimiento y disponibilidad de créditos bancarios, en J.L. Calva, (comp.) Financiamiento del crecimiento económico, UNAM/Porrúa/Cámara de Diputados, México, pp. 19-44.

Lin, J. y R. Tsay. 1996. Cointegration constrain and forecasting: An empirical examination, Journal of Applied Econometrics, 11: 519-538.

Mantey de Anguiano, G. 2007. Política bancaria para el crecimiento con estabilidad, en J.L. Calva, (comp.) Financiamiento del crecimiento económico, UNAM/Porrúa/Cámara de Diputados, México, pp. 45-56.

Martínez, M.A. 1997. "Restricciones de liquidez y consumo en México", Economía: Teoría y Práctica, 8: 105-110. 
McKinnon, R. 1973. Money and capital in economic development, Brookings Institution, Washington.

Mendoza, E. y M. Terrones. 2008. An anatomy of credit booms: Evidence of macro aggregates and firm level data, Board of Governors of the Federal Reserve System (US), International Finance Discussion Papers, num. 936.

Millán, H. 1999. "Las causas de la crisis financiera en México”, Economía, Sociedad y Territorio, II(5): 25-66.

Naka, A. y D. Tufte. 1997. Examining impulse response functions in cointegrated systems, Applied Economics, 29: 1593-1603.

Pagano, M. 1993. Financial markets and growth, an overview, European Economic Review, 37: 613-622.

Palacios, L. 2009. "El carácter rentista y especulativo de la banca mexicana: restricción crediticia y altos márgenes de ganancia”, Economía, 361: 25-40.

Ponce, A. 2003. "Restricciones de liquidez, canal de crédito y consumo en México”, Economía Mexicana, XII(1): 65-101.

Rodríguez, D. y F. López. 2009. "Desarrollo financiero y crecimiento económico en México”, Problemas del Desarrollo, 40(159): 39-60.

Saltz, I. 1999. An examination of the causal relationship between savings and growth in the third world, Journal of Economics and Finance, 23(1): 90-98.

Schumpeter, J. 2003. The theory of economic development, The European Heritage in Economics and the Social Sciences, 1: 61-116 (original, 1911). 
Shaw, E. 1973. Financial deepening in economic development, Oxford University Press.

Sims, C., J. Stock y M. Watson. 1990. Inference in linear time series models with some unit roots, Econometrica, 58: 113-144.Stiglitz, J. 1998.

The role of financial system in development, discurso presentado en The Fourth Annual Bank Conference on Development in Latin America and the Caribbean, El Salvador.

Toda, H. y P. Phillips. 1993. Vector autoregressions and causality, Econometrica, 61: 1367-1393.

Toda, H. e I. Yamamoto. 1995. Statistical inference in vector autoregressions with possibility integrated process, Journal of Econometrics, 66: 225-250.

Tirado, J. 2000. "Crecimiento con cambio tecnológico endógeno, bancos y dinero, El caso de una economía con firmas innovadoras”, Estudios Económicos, 15(1): 91-116.

Velázquez, F. 2006. "Racionamiento del crédito bancario en México y efecto de apalancamiento en las grandes empresas industriales durante el periodo 1995-2004", Economía: Teoría y Práctica. 24: 123-139.

Venegas, F., M.A. Tinoco y V.H. Torres. 2009. "Desregulación financiera, desarrollo del sistema financiero y crecimiento económico en México: efectos de largo plazo y causalidad", Estudios Económicos. 24(2): 249-283. 


\section{APÉNDICE 1}

\section{Cuadro A1 \\ Ecuaciones de cointegración}

Series: IGAE, CREDITO, IGAEPRIM, IGAESEC,CREDCONSUMO

\begin{tabular}{|ccccc|}
\hline Variables & Auto valor & Traza & $5 \%$ & No. de CE(s) \\
IGAE-CREDITO & 27.68168 & 28.7258 & 15.49471 & NINGUNA** \\
& 1.044132 & 1.044132 & 3.841466 & 1 \\
IGAETER-CREDTER & 33.17274 & 36.65215 & 18.39771 & NINGUNA** \\
& 3.479406 & 3.479407 & 3.851466 & 1 \\
IGAE-CREDCONSUMO & 14.32120 & 14.687798 & 15.49471 & NINGUNA** \\
& 0.366589 & 0.366589 & 3.841466 & 1 \\
\hline
\end{tabular}

Nota: ${ }^{* *}$ rechazo de la hipótesis nula a un nivel de significancia de 5\%.

\section{APÉNDICE 2. PRUEBAS DE CAUSALIDAD POR SECTORES}

\section{Cuadro A2}

Causalidad en el sentido de Granger

\begin{tabular}{|llllll|}
\hline Variable dependiente & Crédito & Credprim & Credsec & Credter & Casualidad \\
IGAEPRIM & 0.6342 & 0.7933 & 0.2505 & 0.7215 & No hay casualidad \\
IGAESEC & & 0.695 & 0.7053 & & $\begin{array}{r}\text { No hay casualidad } \\
\text { IGAETER }\end{array}$ \\
\hline & 0.0863 & 0.8112 & 0.9392 & 0.5411 & Crédito $\rightarrow$ IGAETER \\
& $\mathbf{0 . 0 4 7 9}($ VEC) & & & \\
\hline
\end{tabular}

\begin{tabular}{|lcccl|}
\hline Variable dependiente & IGAEPRIM & IGAESEC & IGAETER & Casualidad \\
Crédito & 0.4943 & 0.0337 & 0.1589 & IGAESEC $\rightarrow$ Crédito \\
Credprim & 0.2000 & 0.4789 & 0.2635 & No hay casualidad \\
Credsec & 0.3361 & 0.1038 & 0.5497 & No hay casualidad \\
Credter & 0.0853 & 0.3223 & 0.5411 & No hay casualidad \\
\hline
\end{tabular}




\section{APÉNDICE 3. PRUEBA DE CAUSALIDAD SECTOR TERCIARIO Y SECUNDARIO}

\section{Cuadro A3 \\ Causalidad en el sentido de Granger sector terciario y secundario}

\begin{tabular}{lll|}
\hline $\begin{array}{l}\text { Variable dependiente } \\
\text { IGAETER }\end{array}$ & $\begin{array}{l}\text { IGAESEC } \\
\text { 0.0000 }\end{array}$ & $\begin{array}{l}\text { Credconsumo } \\
\text { 0.0000 }\end{array}$ \\
$\begin{array}{l}\text { Casualidad } \\
\text { IGAESEC } \rightarrow \text { IGAETER } \\
\text { Credconsumo } \rightarrow \text { IGAETER }\end{array}$ & & \\
\hline
\end{tabular}

\begin{tabular}{|lll|}
\hline Variable dependiente & IGAETER & Credconsumo \\
IGAESEC & 0.0000 & 0.1716 \\
Casualidad & & \\
IGAETER $\rightarrow$ IGAESEC & & \\
\hline
\end{tabular}

